\title{
LACK OF COMPACTNESS IN TWO-SCALE CONVERGENCE*
}

\author{
MARC BRIANE ${ }^{\dagger}$ AND JUAN CASADO-DÍAZ
}

\begin{abstract}
This article deals with the links between compensated compactness and two-scale convergence. More precisely, we ask the following question: Is the div-curl compactness assumption sufficient to pass to the limit in a product of two sequences which two-scale converge with respect to the pair of variables $(x, x / \varepsilon)$ ? We reply in the negative. Indeed, the div-curl assumption allows us to control oscillations which are faster than $1 / \varepsilon$ but not the slower ones.
\end{abstract}

Key words. two-scale convergence, compensated compactness, counterexample

AMS subject classifications. 35B27, 35B40

DOI. $10.1137 / 040621351$

1. Introduction. In order to study the asymptotic behavior of periodic problems arising in homogenization theory, Nguetseng introduced in [7] (see also Allaire [1]) the notion of two-scale convergence:

Let $\Omega$ be a bounded open subset of $\mathbb{R}^{d}, Y:=\left(-\frac{1}{2}, \frac{1}{2}\right)^{d}$, and let $M$ be a positive integer. A bounded sequence $u_{\varepsilon}$ in $L_{\mathrm{loc}}^{1}(\Omega)^{M}$ two-scale converges to a function $\hat{u}$ in $L_{\text {loc }}^{1}\left(\Omega \times \mathbb{R}^{d}\right)^{M}$ and $Y$-periodic with respect to the last variable if, for any $\psi \in$ $C_{c}^{\infty}\left(\Omega, C_{\#}^{\infty}(Y)\right)^{M}$, we have

$$
\lim _{\varepsilon \rightarrow 0} \int_{\Omega} u_{\varepsilon}(x) \psi\left(x, \frac{x}{\varepsilon}\right) d x=\int_{\Omega} \int_{Y} \hat{u}(x, y) \psi(x, y) d x d y .
$$

A compactness theorem due to Nguetseng [7] establishes that if $u_{\varepsilon}$ is bounded in $L^{p}(\Omega)^{M}$, then there exists a subsequence of $u_{\varepsilon}$ which two-scale converges to $\hat{u} \in$ $L^{p}\left(\Omega ; L_{\#}^{p}(Y)\right)^{M}$.

Taking in (1.1) $\psi(x, y)$ independent of $y$, we deduce that if $u_{\varepsilon}$ two-scale converges to $\hat{u}$, then it converges weakly in $L^{p}(\Omega)^{M}$ to $u:=\int_{Y} \hat{u}(x, y) d y$. On the other hand, if $u_{\varepsilon}$ strongly converges to $u$ in $L^{1}(\Omega)^{M}$, then it also two-scale converges to $u$. Therefore two-scale convergence is stronger than weak convergence and weaker than the strong one. Moreover, it provides an expression of the limit of the product $u_{\varepsilon} \psi\left(x, \frac{x}{\varepsilon}\right)$ of (1.1) in which each term only weakly converges.

In the periodic homogenization we usually deal with a sequence $u_{\varepsilon}$ which is not only bounded in $L^{p}(\Omega)^{M}$ but whose some combinations of its derivatives are also bounded. In this context, let us recall that if $u_{\varepsilon}$ converges weakly in $W^{1, p}(\Omega)^{M}$, for $1 \leq p<+\infty$, to a function $u$, then it converges strongly in $L_{\mathrm{loc}}^{p}(\Omega)^{M}\left(L^{p}(\Omega)^{M}\right.$ if $\Omega$ smooth) and so $u_{\varepsilon}$ two-scale converges to $u$. Then we can conjecture that the classical results of the compensated compactness theory due to Murat and Tartar (see, e.g., [6] and [8]), and in particular the div-curl theorem, still hold true when we replace the weak convergence in $L^{p}(\Omega)^{M}$ with two-scale convergence. In fact we have the following result:

${ }^{*}$ Received by the editors December 13, 2004; accepted for publication (in revised form) December 28, 2004; published electronically October 14, 2005.

http://www.siam.org/journals/sima/37-2/62135.html

${ }^{\dagger}$ Centre de Mathématiques, I.N.S.A. de Rennes \& I.R.M.A.R., 35043 Rennes cedex, France (mbriane@insa-rennes.fr).

${ }_{\ddagger}^{\ddagger}$ Dpto. de Ecuaciones Diferenciales y Análisis Numérico, Universidad de Sevilla, 41012 Sevilla, Spain (jcasadod@us.es). 
Proposition 1.1. Let $\left(Y, Y_{1}, \ldots, Y_{n}\right)$ be $(n+1)$ parallelotops of $\mathbb{R}^{d}$ of Lebesgue measure equal to 1 , and let $U, V$ be two vector-valued functions in $L^{2}\left(\Omega ; C_{\#}\left(Y \times Y_{1} \times\right.\right.$ $\left.\left.\cdots \times Y_{n}\right)\right)^{d}$, where $C_{\#}\left(Y \times Y_{1} \times \cdots \times Y_{n}\right)$ denotes the set of the continuous functions on $\left(\mathbb{R}^{d}\right)^{n+1}$ which are $Y$-periodic with respect to the variable $y$ and $Y_{k}$-periodic with respect to the variable $y_{k}$ for any $k=1, \ldots, n$. Let $\varepsilon_{k}=\varepsilon_{k}(\varepsilon)$ for $k=1, \ldots, n$ be $n$ well-ordered scales such that

$$
\lim _{\varepsilon \rightarrow 0} \frac{\varepsilon_{1}}{\varepsilon}=\lim _{\varepsilon \rightarrow 0} \frac{\varepsilon_{k+1}}{\varepsilon_{k}}=0 \quad \text { for any } k=1, \ldots, n-1 .
$$

Consider the vector-valued sequences $u_{\varepsilon}$ and $v_{\varepsilon}$ defined by

$$
u_{\varepsilon}(x):=U\left(x, \frac{x}{\varepsilon}, \frac{x}{\varepsilon_{1}}, \ldots, \frac{x}{\varepsilon_{n}}\right) \quad \text { and } \quad v_{\varepsilon}(x):=V\left(x, \frac{x}{\varepsilon}, \frac{x}{\varepsilon_{1}}, \ldots, \frac{x}{\varepsilon_{n}}\right),
$$

and assume that

$$
\operatorname{div} u_{\varepsilon} \text { is compact in } H^{-1}(\Omega) \text { and } \operatorname{curl} v_{\varepsilon} \text { is compact in } H^{-1}(\Omega)^{d \times d} .
$$

Then the two-scale limits $\hat{u}$ of $u_{\varepsilon}, \hat{v}$ of $v_{\varepsilon}$, and $\hat{w}$ of $u_{\varepsilon} \cdot ? v_{\varepsilon}$ exist and satisfy

$$
\hat{w}=\hat{u} \cdot \hat{v}
$$

Proposition 1.1 shows that the div-curl condition (1.4) implies some compactness in the two-scale convergence process (as in the classical case) when the oscillations of the sequences are faster than $\frac{1}{\varepsilon}$. Unfortunately, this is not the case for general sequences, particularly when the oscillations are slower than $\frac{1}{\varepsilon}$. This assertion follows from the following theorem, which is the main result of the present paper:

Theorem 1.2. Assume that $d \geq 2$. Then there exist two functions $U, V \in$ $C_{\#}^{\infty}(2 Y)^{d}$ such that the sequence $u_{\varepsilon}(x):=U\left(\frac{x}{\varepsilon}\right)$ is divergence-free, the sequence $v_{\varepsilon}(x):=V\left(\frac{x}{\varepsilon}\right)$ is curl-free, but the two-scale limits of $u_{\varepsilon}, v_{\varepsilon}$, and $u_{\varepsilon} \cdot v_{\varepsilon}$ do not satisfy (1.5).

The key ingredient of this counterexample is that 2-periodic functions are considered although the test functions are 1-periodic.

In order to understand the lack of compactness in two-scale convergence, let us recall the equivalence between the two-scale convergence theory and the method introduced by Arbogast, Douglas, and Hornung [3] to study the oscillations of a sequence $u_{\varepsilon}$ in $L_{\mathrm{loc}}^{1}\left(\mathbb{R}^{d}\right)^{M}$. Their method consists in introducing the function $\hat{u}_{\varepsilon}$ : $\mathbb{R}^{d} \times Y \rightarrow \mathbb{R}^{M}$ defined by

$$
\hat{u}_{\varepsilon}(x, y)=\sum_{k \in \mathbb{Z}^{d}} 1_{\varepsilon k+\varepsilon Y}(x) u_{\varepsilon}(\varepsilon k+\varepsilon y) .
$$

The equivalence between the two approaches is then given by the following result (see, e.g., [5] and [4]):

THEOREM 1.3. Assume that $u_{\varepsilon}$ is bounded in $L^{p}(\Omega)^{M}$, with $1<p<+\infty$. Then $\hat{u}_{\varepsilon}$ converges weakly to $\hat{u}$ in $L^{p}\left(\Omega ; L^{p}(Y)\right)^{M}$ if and only if $u_{\varepsilon}$ two-scale converges to $\hat{u}$.

The functions $\hat{u}_{\varepsilon}(x, y)$ are not continuous with respect to the variable $x$. If a combination of derivatives of $u_{\varepsilon}$ is bounded, we also get a bound for the same combination of derivatives with respect to the variable $y$ of $\hat{u}_{\varepsilon}$ but not with respect to the variable $x$. This explains the lack of compactness in two-scale convergence. 
2. Proof of the results. In this section we prove Proposition 1.1 and Theorem 1.2 .

Proof of Proposition 1.1. We follow the multiscale procedure of [2]. Thanks to the separation of scales (1.2) the sequences $u_{\varepsilon}, v_{\varepsilon}$, and $u_{\varepsilon} \cdot v_{\varepsilon}$, respectively, twoscale converge to $\hat{u}:=\int_{Y_{1}} \cdots \int_{Y_{n}} U, \hat{v}:=\int_{Y_{1}} \cdots \int_{Y_{n}} V$, and $\hat{w}:=\int_{Y_{1}} \cdots \int_{Y_{n}} U \cdot V$. Putting test functions of type $\varepsilon_{k} \Phi\left(x, \frac{x}{\varepsilon}, \frac{x}{\varepsilon_{1}}, \ldots, \frac{x}{\varepsilon_{k}}\right)$ from $k=n$ to 1 in the div-curl assumption (1.4) implies that

$$
\operatorname{div}_{y_{k}}\left(\int_{Y_{k+1}} \cdots \int_{Y_{n}} U\right)=0 \text { and } \operatorname{curl}_{y_{k}}\left(\int_{Y_{k+1}} \cdots \int_{Y_{n}} V\right)=0 \text { for } k=1, \ldots, n,
$$

whence, integrating by parts the product of $\int_{Y_{k+1}} \cdots \int_{Y_{n}} U$ and $\int_{Y_{k+1}} \cdots \int_{Y_{n}} V$ (which is equal to the gradient in $y_{k}$ of a periodic function plus a function depending only on the other variables $\left.y_{1}, \ldots, y_{k-1}\right)$ successively from $k=n$ to 1 , yields

$$
\hat{w}=\int_{Y_{1}} \cdots \int_{Y_{n}} U \cdot V=\left(\int_{Y_{1}} \cdots \int_{Y_{n}} U\right) \cdot\left(\int_{Y_{1}} \cdots \int_{Y_{n}} V\right)=\hat{u} \cdot \hat{v}
$$

which implies the desired equality (1.5).

Proof of Theorem 1.2. Let us consider two vector-valued functions $\Phi, \Psi \in C_{c}^{\infty}(Y)^{d}$ such that $\operatorname{div} \Phi=0, \operatorname{curl} \Psi=0$, and $\Phi \cdot \Psi \neq 0$ (this is possible since $d>1$ ), which we extend to $\mathbb{R}^{d}$ by $Y$-periodicity. Let $\eta: \mathbb{R} \rightarrow \mathbb{R}$ be the 1-periodic function $\eta:=\sum_{i \in \mathbb{Z}} 1_{\left(i-\frac{1}{4}, i+\frac{1}{4}\right)}$ and let us define the following sequences

$$
u_{\varepsilon}(x):=\eta\left(\frac{x_{1}}{2 \varepsilon}\right) \Phi\left(\frac{x}{\varepsilon}\right) \quad \text { and } \quad v_{\varepsilon}(x):=\eta\left(\frac{x_{1}}{2 \varepsilon}\right) \Psi\left(\frac{x}{\varepsilon}\right) .
$$

Since in each cube $\varepsilon k+\varepsilon Y$, for $k \in \mathbb{Z}^{d}, \eta\left(\frac{x_{1}}{2 \varepsilon}\right)$ is constant, and $\Phi\left(\frac{x}{\varepsilon}\right), \Psi\left(\frac{x}{\varepsilon}\right)$ vanish on the boundary of $\varepsilon k+\varepsilon Y$, we have $u_{\varepsilon}, v_{\varepsilon} \in C^{\infty}\left(\mathbb{R}^{N}\right)$, $\operatorname{div} u_{\varepsilon}=0$, and curl $v_{\varepsilon}=0$ in $\mathbb{R}^{d}$. Moreover, since $\eta\left(\frac{x_{1}}{2 \varepsilon}\right)$ is constant in $\varepsilon k+\varepsilon Y$ for any $k \in \mathbb{Z}^{d}$, it is invariant by the transformation (1.6). So we get

$\hat{u}_{\varepsilon}(x, y)=\eta\left(\frac{x_{1}}{2 \varepsilon}\right) \Phi(y), \quad \hat{v}_{\varepsilon}(x, y)=\eta\left(\frac{x_{1}}{2 \varepsilon}\right) \Psi(y), \quad \widehat{u_{\varepsilon} \cdot v_{\varepsilon}}(x, y)=\eta^{2}\left(\frac{x_{1}}{2 \varepsilon}\right) \Phi(y) \cdot \Psi(y)$.

By Theorem 1.3 the two-scale limits $\hat{u}$ of $u_{\varepsilon}, \hat{v}$ of $v_{\varepsilon}$, and $\hat{w}$ of $u_{\varepsilon} \cdot v_{\varepsilon}$ are thus given by

$$
\begin{gathered}
\hat{u}(x, y)=\left(\int_{-\frac{1}{2}}^{\frac{1}{2}} \eta(s) d s\right) \Phi(y)=\frac{1}{2} \Phi(y), \quad \hat{v}(x, y)=\left(\int_{-\frac{1}{2}}^{\frac{1}{2}} \eta(s) d s\right) \Psi(y)=\frac{1}{2} \Psi(y), \\
\text { and } \hat{w}(x, y)=\left(\int_{-\frac{1}{2}}^{\frac{1}{2}} \eta^{2}(s) d s\right) \Phi(y) \cdot \Psi(y)=\frac{1}{2} \Phi(y) \cdot \Psi(y),
\end{gathered}
$$

whence $\hat{w} \neq \hat{u} \cdot \hat{v}$.

\section{REFERENCES}

[1] G. Allaire, Homogenization and two-scale convergence, SIAM J. Math. Anal., 23 (1992), pp. $1482-1518$.

[2] G. Allaire and M. Briane, Multiscale convergence and reiterated homogenization, Proc. Roy. Soc. Edinburgh A, 126 (1996), pp. 297-342. 
[3] T. Arbogast, J. Douglas, and U. Hornung, Derivation of the double porosity model of single phase flow via homogenization theory, SIAM J. Math. Anal., 21 (1990), pp. 823-836.

[4] D. Cioranescu, A. Damlamian, and G. Griso, Periodic unfolding and homogenization, C. R. Acad. Sci. Paris, 335 (2002), pp. 99-104.

[5] M. Lenczner, Homogénéisation d'un circuit électrique, C. R. Acad. Sci. Paris, 324 (1997), pp. $537-542$.

[6] F. Murat, Compacité par compensation, Ann. Scuola Norm. Sup. Pisa Cl. Sci., 5 (1978), pp. $489-$ 507.

[7] G. NGuetseng, A general convergence result for a functional related to the theory of homogenization, SIAM J. Math. Anal., 20 (1989), pp. 608-623.

[8] L. TARTAR, Compensated compactness and applications to partial differential equations, nonlinear analysis and mechanics, in Heriot-Watt Symposium IV, Pitman, San Francisco, 1979, pp. $136-212$. 